\title{
1 Construction of redesigned pMAL expression vector for 2 easy and fast purification of active native antimicrobial 3 peptides
}

4

5 Lazar Gardijan ${ }^{1}$, Marija Miljkovic ${ }^{1}$, Mina Obradovic ${ }^{1}$, Branka Borovic $^{2}$, Goran Vukotic ${ }^{1,3}$,

6 Goran Jovanovic ${ }^{1}$, and Milan Kojic ${ }^{1 *}$

$7{ }^{1}$ Laboratory for Molecular Microbiology, Institute of Molecular Genetics and Genetic

8 Engineering, University of Belgrade, Vojvode Stepe 444/a, 11042 Belgrade, Serbia;

9 2Institute of Meat Hygiene and Technology, Kaćanskog 13, 11040 Belgrade, Serbia

$10 \quad{ }^{3}$ Faculty of Biology, University of Belgrade, Studentski trg 16, 11158 Belgrade, Serbia

11 \#Authors contributed equally

12

13

$14{ }^{*}$ Corresponding author:

15 Milan Kojic

16 e-mail:mkojic@imgge.bg.ac.rs

17 Tel.: +381 113975960

18 orcid.org/0000-0001-5645-750X 


\section{Abstract}

21 Many protein expression and purification systems are commercially available to provide a sufficient amount of pure, soluble and active native protein, such as the pMAL system based on E. coli maltose binding protein tag (MBP). Adding specific amino acid tags to the $\mathrm{N}$ - or $\mathrm{C}$ terminus of the protein increases solubility and facilitates affinity purification of proteins. However, many of expressed tagged proteins consequently lose functionality, particularly small peptides such as antimicrobial peptides (AMPs). Objective of this study was to redesign the pMAL expression vector in order to increase the efficacy of MBP tag separation from native peptides. Redesign of the pMAL expression vector included introduction of the $\mathrm{His}_{6}$ tag and the enterokinase cleavage site downstream from the original MBP tag and Xa cleavage site enabling purification of native and active peptide $(\mathrm{P})$ following two-step affinity chromatography. In the first step the entire MBP-His 6 -P fusion protein is purified through binding to Ni-NTA agarose. In the second step, the purification was performed by adding mixture of amylose and Ni-NTA agarose resins following cleavage of the fusion protein with active $\mathrm{His}_{6}$ tagged enterokinase. This removes MBP-His 6 and $\mathrm{His}_{6}$-enterokinase leaving pure native protein in solution. The redesigned pMAL vectors were optimized for cytoplasmic (pMALc5HisEk) and periplasmic (pMALp5HisEk) peptides expression. Two-step purification protocol was successfully applied in purification of active native AMPs, lactococcin A and human $\beta$-defensin. Taken together, we established the optimal conditions and pipeline for overexpression and purification of large amount of native peptides, that can be implemented in any laboratory.

Keywords: pMALc5HisEk vector, pMALp5HisEk vector, pQE_Ek vector, His ${ }_{6}$-enterokinase cleavage, two-step purification, removal of tags, antimicrobial peptides (AMPs). 


\section{Introduction}

Proteins play crucial and diverse roles in all organisms. They represent the building blocks of every cell, act as enzymes, hormones, cell attachment anchors, regulators and source of amino acids, play role in signaling and defense as antibodies or antimicrobial peptides (AMPs), and contribute in many other processes. The protein over-production and purification is a powerful tool in biotechnology and basic science research and need for a variety of purified native proteins is elevated over the last decade $[1,2]$. However, the biotechnology industry is still faced with insufficient production capacity due to increasing demand for biologically active proteins and in particular those used as therapeutics [3]. The favorite host for recombinant protein production is an Escherichia coli, not only for ability to grow rapidly and achieve high density on cheap media, but also because of well-characterized genetics and the available number of cloning vectors and mutant host strains [4-6]. There are many protein expression and purification systems developed based on E. coli host and the corresponding expression vectors. Majority of expression vectors are designed to introduce the amino acids tag to the protein of interest leading to expression of the tagged fusion proteins [6-8]. Many of those fusion proteins retain the activity close to wild type, even in the presence of the tag. However, a significant number of tagged proteins lose the activity and so require to be purified in its native form. This is particularly true for the smaller proteins such as peptides, or more specifically, the antimicrobial peptides (AMPs). Importantly, AMPs are at the forefront of the effort to replace/substitute the antibiotics and tackle the ever increasing antimicrobial resistance problem [9-11].

In this work, we redesigned the pMAL vector which is part of the well known protein expression and purification system typically used to purify insoluble proteins [12-14]. Here, the relatively large size maltose binding protein (MBP) of $\sim 43 \mathrm{kDa}$ is fused as a tag to the protein of interest and the native protein is purified following overexpression and purification through amylose 
69 resin column and cleavage by Xa protease which cleavage site is positioned between the MBP

70 and the protein. This system is designed to require the use of the size exclusion columns/filters

71 to separate the many smaller proteins from MBP. However, if the native protein of interest is

72 too small, chemical characteristics of the size exclusion columns/filters, cause non-specific

73 binding of the small peptide to the column. Therefore, the rational to redesign the pMAL vector

74 and system was to accommodate the vector and develop the protocol for overexpression and

75 purification of the small peptides, specifically AMPs. The effectiveness of novel system was

76 tested using well characterized AMPs, lactococcin A (LcnA) and human $\beta$-defensin (hBD). 


\section{Materials and Methods}

\section{Bacterial strains and growth conditions}

Escherichia coli strains DH5 $\alpha$ [15] and ER2523 (New England Biolabs, Ltd., UK) were grown in Luria Bertani (LB) medium at $37^{\circ} \mathrm{C}$ with aeration (180 rpm). Lactococcus lactis BGMN1596 was grown in M17 medium (Merck GmbH, Darmstadt, Germany) supplemented with Dglucose $(0.5 \% \mathrm{w} / \mathrm{v})(\mathrm{GM} 17)$ at $30^{\circ} \mathrm{C}[16]$. Solid medium and soft agar were made by adding $1.5 \%$ and $0.7 \%(\mathrm{w} / \mathrm{v})$ agar (Torlak, Belgrade, Serbia) to the liquid media, respectively. Ampicillin $(100 \mu \mathrm{g} / \mathrm{ml})$ was used for selection and maintaining of transformants. Isopropyl- $\beta$ D-1-thiogalactopyranoside (IPTG; Serva, Heidelberg, Germany) in appropriate concentrations was used for the induction of protein expression.

\section{DNA manipulations}

For plasmid isolation from E. coli transformants, a Thermo Fisher Scientific GeneJET Plasmid Miniprep kit was used according to the manufacturer's recommendations (Thermo Scientific, Lithuania). Digestion with restriction enzymes was conducted according to the supplier's instructions (Thermo Fisher Scientific Waltham, MA, USA). DNA was ligated using T4 DNA ligase (Agilent technologies, USA) according to the manufacturer's recommendations. Standard heat-shock transformation method was used for transformation of $E$. coli with a plasmid [15].

\section{Site-directed mutagenesis}

Desired mutations were introduced as described previously by Miljkovic et al. [17] using oligonucleotide primers (and their reverse complements) carrying mutations (see Table 1). PCR amplification of each plasmid DNA strand for site-directed mutagenesis was performed separately by adding only one primer. Amplification was conducted by Phusion High Fidelity DNA Polymerase (Thermo Fisher Scientific) one $\mathrm{min} / \mathrm{kb}$. Single strand amplicons obtained by 
102 forward and complement primers were annealed (see below) and digested with $1 \mu 1(10 \mathrm{U})$ of

$103 D p n I$ restriction enzyme per reaction (at $37^{\circ} \mathrm{C}$ for $2 \mathrm{~h}$ ) to destroy the methylated template

104 strands. Non-digested plasmid DNA was used for transformation of E. coli DH5 $\alpha$ high-

105 competent cells by heat shock treatment. Transformants were selected on LA Petri dishes

106 containing ampicillin. To obtain plasmid DNA from the selected colonies, the GeneJET plasmid

107 miniprep kit was used according to the manufacturer's recommendations (Thermo Fisher

108 Scientific). The introduction of the desired mutations was confirmed by sequencing (Macrogen

109 Europe, The Netherlands).

110 Table 1. Primers and olignucleotides used in this study.

\begin{tabular}{|c|c|}
\hline $\begin{array}{l}\text { Name of primer/ } \\
\text { oligonucleotide }\end{array}$ & Sequence $5^{\prime}-3^{\prime}$ \\
\hline BsaBI-2199-F & CAGCGATGACCAT $\underline{\text { TAACGGCCCGTG }}$ \\
\hline BsaBI-2199-R & CCGTTA_ATGGTCATCGCTGTTTCGCC \\
\hline BsaBI-5422-F & CCTGCGATGCAGAĆCCGGAACATAATGG \\
\hline BsaBI-5422-R & GTTCCGGGTCTGCATCGCAGGATGCTGC \\
\hline 6xHis_BsaBI-Ek-F & $\begin{array}{l}\text { CATCACCATCACCATCACGGATCTGATGATGATGATAATTATCGCATGCG } \\
\text { AGCTCGGTACCCC }\end{array}$ \\
\hline 6xHis_BsaBI-Ek-R & $\begin{array}{l}\text { GGGGTACCGAGCTCGCATGCGATAATTATCATCATCATCAGATCCGTGAT } \\
\text { GGTGATGGTGATG }\end{array}$ \\
\hline BsaBI-Ek-pQE-F & GATCTGATGATGATGATAATTATCGCATGCGAGCTCGGTACCCC \\
\hline BsaBI-Ek-pQE-R & GGGGTACCGAGCTCGCATGCGATAATTATCATCATCATCA \\
\hline hBD-BH_F_Ek-111 & $\begin{array}{l}\text { AGATCATTATAACTGCGTGTCGTCGGGCGGCCAGTGCCTGTATTCGGCGT } \\
\text { GCCCGATTTTTACCAAAATTCAGGGCACCTGCTATCGCGGCAAAGCGAAA } \\
\text { TGCTGCAAATA }\end{array}$ \\
\hline hBD-BH_R_Ek-115 & $\begin{array}{l}\text { AGCTTATTTGCAGCATTTCGCTTTGCCGCGATAGCAGGTGCCCTGAATTT } \\
\text { TGGTAAAAATCGGGCACGCCGAATACAGGCACTGGCCGCCCGACGACACG }\end{array}$ \\
\hline
\end{tabular}




\begin{tabular}{|c|c|}
\hline & CAGTTATAATGATCT \\
\hline LcnA-BH_F_Ek-166 & $\begin{array}{l}\text { AAAATTAACATTTATTCAATCGACAGCGGCTGGAGATTTATATTACAATA } \\
\text { CTAATACACACAAATATGTTTACCAACAAACTCAAAACGCTTTTGGGGCT } \\
\text { GCTGCTAATACCATTGTTAATGGATGGATGGGTGGCGCTGCTGGAGGTTT } \\
\text { CGGGTTGCACCATTGA }\end{array}$ \\
\hline LcnA-BH_R_Ek-170 & $\begin{array}{l}\text { AGCTTCAATGGTGCAACCCGAAACCTCCAGCAGCGCCACCCATCCATCCA } \\
\text { TTAACAATGGTATTAGCAGCAGCCCCAAAAGCGTTTTGAGTTTGTTGGTA } \\
\text { АACATATTTGTGTGTATTAGTATTGTAATATAAATCTCCAGCCGCTGTCG } \\
\text { АTTGAATAAATGTTAАTTTT }\end{array}$ \\
\hline
\end{tabular}

111 The single nucleotides mutated within oligonucleotides used for site directed mutagenesis are

112 indicated by bold and underlined letters.

\section{Annealing of DNA oligonucleotides}

115 Annealing of two single-stranded DNA oligonucleotides with complementary sequences was

116 done by heating and cooling method. Oligonucleotides were dissolved in pure water. Annealing

117 mixture $(50 \mu 1)$ composed of two oligonucleotides (with the equimolar concentration $10 \mu \mathrm{M}$ )

118 in annealing buffer (10 mM Tris-HCl-pH 7.5, $50 \mathrm{mM} \mathrm{NaCl}, 1 \mathrm{mM}$ EDTA) was incubated for 5

119 min at $95-100^{\circ} \mathrm{C}$ and after that slowly cool down to room temperature before transferring it on

120 ice. Hybridized oligonucleotides were used for ligation with predigested vectors or stored at -

$12120^{\circ} \mathrm{C}$.

122 Overexpression and two-step purification of the recombinant AMPs in E. coli ER2523

123 In this study we tested the efficacy of newly constructed (reconstructed) pMAL expression

124 vectors pMALc5HisEk and pMALp5HisEk by expressing and purifying two AMPs, through

125 two-step purification. We used Ni-NTA agarose and amylose resins affinity chromatographies

126 in two different combinations. Transformants of E. coli ER2523 were maintained overnight on

127 LA Petri dishes containing ampicillin $(100 \mu \mathrm{g} / \mathrm{ml})$ and glucose $(1 \%)$ at $30^{\circ} \mathrm{C}$. The next day new 
cultures (each $200 \mathrm{ml}$ of LB with $1 \%$ glucose) were inoculated using $2 \%$ sample of overnight culture and incubated at $30^{\circ} \mathrm{C}$ with aeration $(180 \mathrm{rpm}$ on rotatory shaker). Expression of recombinant peptides was carried out in LB containing ampicillin $(100 \mu \mathrm{g} / \mathrm{ml})$ and glucose $(1 \%)$

131 and protein production was induced in logarithmic growth phase $\left(\mathrm{OD}_{600}=0.8-1.0\right)$ with addition

132 of $0.3 \mathrm{mM}$ (final) IPTG for $3 \mathrm{~h}$. Bacterial cells were collected by centrifugation at $4500 \mathrm{x} g$ and

133 before purification the level of induction was tested by comparing the amount of total proteins

134 from the same amount of induced and non-induced cells. Purification including cell lysis,

135 affinity chromatographies, and cleavage of the fusion protein with enterokinase that were

136 performed according to manufacturer's instructions (amylose resin purification - according to

137 pMAL Protein Fusion \& Purification System, New England Biolabs, Ltd., UK; Ni-NTA agarose

138 resin purification - according to The QIAexpressionist, Qiagen Gmbh, Hilden, Germany;

139 cleavage with recombinant bovine enterokinase - according to GenScript, New Jersey, USA),

140 with the addition of cell lysis step that was performed in column buffer (CB) with $1 \mathrm{mg} / \mathrm{ml}$

141 lysozyme for $30 \mathrm{~min}$ on ice.

142 Firstly, the Ni-NTA agarose resin purification step was performed and then purified fusion 143 protein was digested in $1 \mathrm{X}$ enterokinase buffer $(20 \mathrm{mM}$ Tris- $\mathrm{HCl}, \mathrm{pH}$ 7.4, $50 \mathrm{mM} \mathrm{NaCl}, 2 \mathrm{mM}$

$144 \mathrm{CaCl}_{2}$ ) using $10 \mathrm{U}$ of Recombinant Bovine His 6 -Enterokinase (GenScript, Piscataway, NJ,

145 USA) overnight at room temperature. In order to place the purified protein in $1 \mathrm{X}$ enterokinase

146 buffer, the buffer exchange was done using Amicon Ultra $0.5 \mathrm{ml} 30 \mathrm{~K}$ Centrifugal filters (Merck

147 Millipore Ltd, Cork, Ireland). After $24 \mathrm{~h}$ of digestion, total cleavage of the purified protein was

148 obtained and the second purification step using amylose and Ni-NTA agarose resins mix was

149 performed according to the instructions (The QIAexpressionist).

150 Proteins from every step of expression, purification and proteolysis were analyzed on $12.5 \%$

151 sodium dodecyl sulfate polyacrylamide gel electrophoresis (SDS-PAGE). Samples for SDS-

152 PAGE were mixed with $2 \mathrm{X}$ sample loading buffer (125 mM TrisHCl pH6.8, $10 \mathrm{mM}$ EDTA, 
A spot-on-the-lawn inhibition assay was used for testing antimicrobial activity of purified recombinant AMPs as previously described [18]. L. lactis BGMN1-596 [16] was used as sensitive strain in antimicrobial assay. $5 \mu$ of the purified AMP was applied to soft agar inoculated with L. lactis BGMN-596. The presence of inhibition zones was examined after 24 antimicrobial peptide activity. The antimicrobial activity assay was performed in at least two

162 independent experiments. The enterokinase buffer (in which the purified AMPs were resuspended) was taken as a negative control in an antimicrobial assay.

\section{Results and Discussion}

\section{Rational for redesigning pMAL expression vector}

167 In our previous work the most successful expression and purification of AMPs, was achieved with the pMAL expression and purification system [19-21]. The pMAL expression vector (New

169 England Biolabs) provided a high yield of soluble native peptide following precise excision of MBP tag using Xa protease. However, we encountered the problem in separating the MBP tag

171 from dissolved purified low molecular weight AMPs. We tried to facilitate the process by

172 passing digested eluates through a $10 \mathrm{kDa}$ cut-off column, but the yield was not sufficient due

173 to the high binding of the small peptide to the column, even though the low-binding columns

174 were used.

175 To meet the requirements for the fast and simple separation of expressed AMPs from the MBP

176 tag we decided to design and generate an improved version of pMAL expression vector, but 177 different from the one already available pDEST-HisMBP (www.addgene.org, plasmids 
\#11085). pDEST-HisMBP contains the $\mathrm{His}_{6}$ tag positioned at the MBP N-terminus and a TEV protease recognition site at $\mathrm{C}$-terminus for the excision of the recombinant peptide [22]. There is a commercially available $\mathrm{His}_{6}$ tagged enterokinase protease that cuts immediately after the

181 recognition cleavage site potentially releasing precisely the expressed native peptide. The idea

182 was to use pMAL vector and introduce the $\mathrm{His}_{6}$ tag and the enterokinase cleavage site

183 downstream from the MBP and Xa cleavage site and just upstream of the Multi Cloning Site

184 (MCS) used for cloning the desired peptide DNA (Fig 1). This will provide the effective affinity separation of the MBP-His 6 tag and the $\mathrm{His}_{6}$-enterokinase from the peptide following cleavage of the composite MBP-His ${ }_{6}$-Ek-P fusion protein with the His $_{6}$-enterokinase and use of Ni-NTA agarose.

Fig 1. Construction of redesigned pMAL and pQE vectors. Circular maps and depicted redesigned nucleotide sequence of (A) pMALc/p5HisEk and (B) pQE_Ek vectors. Arrows

191 indicate the size and direction of gene transcription. Unique restriction sites are indicated by

192 bold letters. malE: maltose binding protein (MBP) gene, Xa: Xa factor cleavage site IEGR

193 (typical Xa cleavage site is IE/DGR), 6xHis: 6xHis tag sequence, iEk: incomplete enterokinase

194 (Ek) cleavage site (DDDDN) to be reconstituted into complete Ek cleavage site DDDDK 195 following cloning, MCS: multiple cloning site with restriction sites indicated, bla: ampicillin 196 resistance gene, $l a c I, l_{a c I}{ }^{\mathrm{a}}$ repressor gene, Ptac: taq promoter, pBRori: pBR322 origin of 197 replication, PT5: T5 promoter, lacO: lac operator, RBS: ribosome-binding site, ATG: start 198 codon, Stop Codons: stop codons in all three reading frames, Col E1: ColE1 origin of 199 replication. 
202 We constructed redesigned pMAL expression vectors pMALc5HisEk and pMALp5HisEk 203 based on pMALc5X (for expression of proteins in the cytoplasm; New England Biolabs) and 204 pMALp5X (for expression of proteins in the periplasm; New England Biolabs). Namely, the 205 MBP at the N-terminus in pMALp5X contains the leader peptide for transfer of synthesized 206 protein to the periplasm. The restriction enzyme recognition site sequence analysis showed that 207 the best restriction enzyme DNA sequence that can form the enterokinase recognition site (DDDDK) and be placed upstream of the $5^{\prime}$ end from the MCS is $B s a \mathrm{BI}$ (GATNN/NNATC) (see Fig 1). The choice of $B s a \mathrm{BI}$ restriction enzyme was further supported by the fact that it is a very efficient restriction enzyme that cuts DNA at $60-65^{\circ} \mathrm{C}$, with no star activity, introducing

211 blunt ends. However, the sequence analysis of pMALc5X and pMALp5X vectors revealed that 212 they possess two BsaBI restriction sites; one in the malE gene (position 2199c/2273p, respectively) and the other at position $5422 \mathrm{c} / 5497 \mathrm{p}$, respectively). To destroy these sites so that

214 the BsaBI restriction site could be introduced upstream of the multi cloning site for cloning 215 DNA fragments, the site-specific mutagenesis of both $B s a \mathrm{BI}$ restriction sites in pMAL vectors 216 was done using the primer pairs listed in Table 1. Since BsaBI restriction sites are mapped in 217 coding genes, vital for plasmid maintenance and expression, the mutations introduced by site 218 directed mutagenesis (the codons showing the highest frequency in E. coli were selected) 219 formed the same sense codons. The success of the restriction site mutagenesis was first checked 220 by digestion with $B s a \mathrm{BI}$ restriction enzyme, and finally confirmed by sequencing. Before 221 proceeding to further construction, the functionality of the mutated pMAL vectors 222 (pMALc5X $\Delta$ BsaBI and pMALp5X $\Delta$ BsaBI) was checked. We induced the MBP expression 223 using IPTG and detected the production of overexpressed MBP protein with the correct 224 molecular weight on PAGE SDS electrophoresis.

225 The $\mathrm{His}_{6}$ tag and the $B s a \mathrm{BI}$ restriction site were introduced upstream of the multi cloning site 226 of either pMALc5X $\Delta$ BsaBI or pMALp5X $\Delta$ BsaBI using oligonucleotides $6 x$ His_BsaBI-Ek-F 
and 6xHis_BsaBI-Ek-R (see Table 1). After annealing, double strand hybrid DNA fragment

228 was inserted into the $X m n \mathrm{I}$ restriction site. In addition to $B s a \mathrm{BI}$ site, oligonucleotides were designed to introduce an extra $S a c$ I restriction enzyme recognition site downstream from $B s a \mathrm{BI}$

230 (Fig 1A). The SacI restriction enzyme digestion was used to check for the correct orientation of 231 the inserted DNA fragment. The plasmid DNA from transformants was isolated and digested 232 with the SacI restriction enzyme. Clones that produced a fragment of 104 bps were selected 233 because they contain a cloned fragment in the right orientation. This was confirmed by 234 sequencing. We intentionally introduced the incomplete enterokinase recognition site (iEk:

235 DDDDN instead of complete one DDDDK) being able to manipulate the N nucleotide in $B s a \mathrm{BI}$ 236 restriction site [introducing asparagine $(\mathrm{N})$ instead of Lysine $(\mathrm{K})$ ] (see Fig 1A). The reason for 237 this is to emphasize need to add an extra adenine to the $5^{\prime}$ end of the desired protein/peptide 238 coding sequence (or to the forward primer used for amplification of desired protein/peptide 239 coding sequence) in order to obtain both, in frame protein/peptide synthesis with MBP tag and 240 the constitution of complete enterokinase cleavage site (DDDDK). Two newly constructed 241 expression vectors were named pMALc5HisEk and pMALp5HisEk. The inducibility and 242 production of overexpressed ${\mathrm{MBP}-H i s_{6}}_{6}$ from the redesigned pMAL vectors was checked 243 following induction with $0.3 \mathrm{mM}$ IPTG for 3 hours, and so the production of correct fusion $244 \mathrm{MBP}-\mathrm{His}_{6}$ protein was obtained (Fig 2A).

246 Fig 2. Expression quality control from pMALc5HisEk and pMALp5HisEk vectors. SDS-

247 PAGE analysis of (A) ${\mathrm{MBP}-H i s_{6}}_{6}$ expression in E. coli $\mathrm{DH} 5 \alpha$ and (B) recombinant fusion 248 proteins MBP-His 6 -LcnA and MBP-His ${ }_{6}$-hBD expression in E. coli ER2523, before and after 249 induction. (A) Total proteins of E. coli DH5 $\alpha$ carrying pMALc5HisEk or pMALp5HisEk 250 before (lane 1 and 3) and after induction with $0.3 \mathrm{mM}$ IPTG (lane 2 and 4). Note a significant

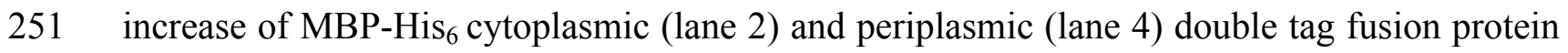


expression ( $\sim 3 \mathrm{kDa}$; indicated by black arrow). (B) Total proteins of E. coli ER2523 carrying pMALc5HisEk_LcnA1 or pMALp5HisEk_hBD1 before (lane 1 and 3) and after induction with protein $\mathrm{MBP}_{-\mathrm{His}_{6}}$-LcnA (lane 2; $\sim 50 \mathrm{kDa}$; ) and periplasmic fusion protein MBP-His ${ }_{6}-\mathrm{hBD}$ (lane 4, $\sim 50 \mathrm{kDa}$ ), indicated by black arrow. Lane M: protein molecular weight marker (BlueEasy Prestained Protein Marker)

In addition, we constructed pQE_Ek expression vector for the N-terminal peptide fusions following the same strategy and using BsaBI-Ek-pQE-F and BsaBI-Ek-pQE-R oligonucleotides including BsaBI restriction site into pQE30 previously digested with BamHI (Fig 1B).

Optimization of expression and purification of LenA and hBD AMPs using redesigned

To demonstrate the efficacy of redesigned pMAL vectors pMALc5HisEk and pMALp5HisEk, two AMPs were selected for overexpression, purification and functionality check: lactococcin A (LcnA) for cytoplasmic expression and human beta defensin (hBD) for periplasmic expression, to allow a formation of disulfide bridges.

269 The coding sequences for LcnA and hBD were synthetic and obtained by annealing of corresponding oligonucleotides (Table 1). The codon selection for hBD was optimized for

271 codon usage of E. coli. Extra adenine (A) were added upstream of coding sequences for both 272 AMPs while $3^{\prime}$ ends contained the sticky ends compatible with HindIII in digested vectors 273 (Table 1). The dsDNA fragments obtained by annealing of corresponding oligonucleotides were 274 cloned into BsaBI-HindIII pre-digested pMALc5HisEk or pMALp5HisEk vectors. More than $27590 \%$ of analyzed transformants contained plasmid carrying the desired DNA fragment. We 276 sequenced 5 clones from each transformation using pMalE forward primer to confirm the 
orientation, in frame position with the MBP-His 6 tags, and the presence of the correct enterokinase cleavage recognition site. The newly generated pMALc5HisEk_LcnA1 and pMALp5HisEk_hBD1 constructs were stored at $-80^{\circ} \mathrm{C}$ in $\mathrm{LB}$ containing $15 \%$ glycerol and further used for the expression and purification experiments.

For overexpression of LcnA and hBD AMPs, the E. coli strain ER2523 was transformed with pMALc5HisEk_LcnA1 or pMALp5HisEk_hBD1 and grown overnight at $30^{\circ} \mathrm{C}$. Next day the production of AMPs was induced in the logarithmic phase of growth and the level of induction was tested comparing the amount of total proteins from the same amount of induced and noninduced cells (Fig 2B).

Next, we demonstrated that both tags are individually efficient/active, enabling purification of overexpressed MBP-His 6 -LcnA and MBP-His 6 -hBD. Induced cultures were divided into two 288 equal aliquots and expressed fusion proteins were purified either via amylose resin (binding MBP-His ${ }_{6}$-AMP fusion protein, followed by cleavage with enterokinase, and incubation with

Ni-NTA agarose resin in the second step to remove the MBP-His 6 and $\mathrm{His}_{6}$-enterokinase from solution, leaving native AMP purified. However, there was no binding of the MBP-His 6 to the 
303 Lane 1: eluted fusion protein MBP-His ${ }_{6}-\mathrm{LcnA}$ using amylose resin, (B) Lane 1: eluted fusion second step purification.

In the second strategy/attempt, the splitting of MBP-His ${ }_{6}$ and AMP peptide was performed by overnight digestion of AMP with $\mathrm{His}_{6}$-enterokinase at room temperature following purification

313 of using either MBP or His tag, (see Materials and Methods). We noted that the efficiency of

314 the protease cleavage was much higher when protein was isolated using Ni-NTA agarose (S3

315 Fig, line 3), although the proteins were completely digested after $24 \mathrm{~h}$, differences were 316 observed at earlier time points. This might be the consequence related to difference in the availability of the enterokinase cleavage site present on the original MBP-His 6 -Ek-P fusion 318 protein that has undergone conformational changes due to maltose binding. The removal of 319 MBP-His 6 and $\mathrm{His}_{6}$-enterokinase was accomplished in second step purification using a mix of 320 amylose (to bind $\mathrm{MBP}^{-\mathrm{His}_{6}}$ tag) and Ni-NTA agarose (to bind $\mathrm{His}_{6}$-enterokinase) in a ratio of 321 10:1 (Fig 4) only from digestion of a fusion protein purified using Ni-NTA agarose in first step.

322 The expressed AMPs, LcnA (Fig 4A, line 5) and hBD (Fig 4B, line 5) were isolated in over $32390 \%$ purity as shown when compared to the sample obtained after cleavage with enterokinase 324 (Fig 4A and 4B, lane 4). 

of Ni-NTA agarose and amylose : Ni-NTA agarose (10:1) resins mix. SDS-PAGE analysis of two-step protein purification of (A) LcnA and (B) hBD AMPs using first Ni-NTA agarose and then 10:1 mix of amylose and Ni-NTA agarose resins. Lane 1: total proteins of E. coli ER2523 carrying (A) pMALc5HisEk_LcnA1 and (B) pMALp5HisEk_hBD1 before induction, Lane 2: after induction with $0.3 \mathrm{mM}$ IPTG (A and B) and expression of $\sim 50 \mathrm{kDa}$ fusion protein, with Ni-NTA agarose resin, Lane 4: products obtained after cleavage with $\mathrm{His}_{6}$-enterokinase which splits fusion protein into MBP-His ${ }_{6}$ tag of $\sim 43 \mathrm{kDa}$ and expressed $\sim 5 \mathrm{kDa}$ AMP (A) after the second step of affinity chromatography purification using 10:1 mix of amylose : NiNTA agarose resins. Lane M: protein molecular weight marker (BlueEasy Prestained Protein Marker). Black arrows indicate the products of cleavage by $\mathrm{His}_{6}$-enterokinase. Open arrow indicates the position of the $\mathrm{His}_{6}$-entrokinase on the gel.

341 The functionality/bioactivity of the purified native LcnA and hBD was analysed by spot on the

342 lawn antimicrobial assay using L. lactis BGMN-596 sensitive strain. Both purified native AMPs 343 showed an antimicrobial activity (Fig 5). The activity of LcnA was higher than hBD and this 344 might be the consequence of incomplete folding of the hBD within hybrid protein in the periplasm or used indicator strain is less sensitive to $\mathrm{hBD}$.

Fig 5. The antimicrobial activity of purified LenA and hBD. LcnA and hBD were expressed 348 in E. coli ER2523 (using pMAL-c/p5HisEk vectors), purified and spotted (5 $\mu$ l) on L. lactis 349 BGMN1-596 sensitive strain. LcnA; lactococcin A, hBD: human beta defensin, buffer: $5 \mu 1$ of enterokinase buffer (negative control). 


\section{Conclusions}

352 Here we report an efficient procedure for separation of native peptides from the tag(s), using 353 redesigned pMAL expression vector and the two-step affinity chromatography. The idea of 354 redesigning pMAL vector and creating a novel two-tag expression vector(s) was born from the need for rapid purification of larger amounts of active AMPs. We constructed novel pMAL356 based vectors, pMALc5HisEk and pMALp5HisEk, which enable purification of active native 357 AMPs from the cytoplasm and periplasm, and demonstrated validity of the novel protocol using LcnA and hBD AMPs. This system is intended for the cloning, expression and purification of heterologous proteins/peptides in E. coli and includes fast and reliable procedure for the isolation of active and native recombinant proteins and peptides (Fig 6). By applying this expression and purification protocol, protein purity is greater than $90 \%$, thanks to a double tag separation and protease removal. Moreover, this system is simple and cost-effective, and can be implemented in any laboratory.

Fig 6. Flow chart presenting pipeline for cloning, expression and two-step purification of peptides/AMPs using redesigned pMAL-c/p5HisEk vectors in E. coli ER2523. X: any unique restriction enzyme from the multi cloning site absent from the peptide gene.

\section{Acknowledgements and Funding}

372 This work was supported by The Ministry of Education, Science and Technological

373 Development of the Republic of Serbia, Republic of Serbia (Grant No.173019). 


\section{References}

1. Vecchio I, Tornali C, Bragazzi NL, Martini M. The Discovery of Insulin: An Important Milestone in the History of Medicine. Front Endocrinol (Lausanne). 2018;9:613. doi: 10.3389/fendo.2018.00613. PMID: 30405529; PMCID: PMC6205949.

2. Geddes BA, Mendoza-Suárez MA, Poole PS. A Bacterial Expression Vector Archive (BEVA) for Flexible Modular Assembly of Golden Gate-Compatible Vectors. Front Microbiol. 2019;9:3345. doi: 10.3389/fmicb.2018.03345. PMID: 30692983; PMCID: PMC6339899.

3. Mergulhao FJM, Monteiro GA, Cabral JMS, Taipa MA. Design of bacterial vector systems for the production of recombinant proteins in Escherichia coli. J Microbiol Biotechnol. 2004; $14(1), 1-14$.

4. Structural Genomics Consortium; China Structural Genomics Consortium; Northeast Structural Genomics Consortium, Gräslund S, Nordlund P, Weigelt J, Hallberg BM, Bray J, Gileadi O, Knapp S, Oppermann U, Arrowsmith C, Hui R, Ming J, dhe-Paganon S, Park HW, Savchenko A, Yee A, Edwards A, Vincentelli R, Cambillau C, Kim R, Kim SH, Rao Z, Shi Y, Terwilliger TC, Kim CY, Hung LW, Waldo GS, Peleg Y, Albeck S, Unger T, Dym O, Prilusky J, Sussman JL, Stevens RC, Lesley SA, Wilson IA, Joachimiak A, Collart F, Dementieva I, Donnelly MI, Eschenfeldt WH, Kim Y, Stols L, Wu R, Zhou M, Burley SK, Emtage JS, Sauder JM, Thompson D, Bain K, Luz J, Gheyi T, Zhang F, Atwell S, Almo SC, Bonanno JB, Fiser A, Swaminathan S, Studier FW, Chance MR, Sali A, Acton TB, Xiao R, Zhao L, Ma LC, Hunt JF, Tong L, Cunningham K, Inouye M, Anderson S, Janjua H, Shastry R, Ho CK, Wang D, Wang H, Jiang M, Montelione GT, Stuart DI, Owens RJ, Daenke S, Schütz A, Heinemann U, Yokoyama S, Büssow K, Gunsalus KC. Protein production and purification. Nat Methods. 
401

402

403

404

405

406

407

408

409

410

411

412

413

414

415

416

417

418

419

420

421

422

2008;5(2):135-46. doi: 10.1038/nmeth.f.202. Erratum in: Nat Methods. 2008;5(4):369.

Hallberg, B Martin [added]. PMID: 18235434; PMCID: PMC3178102.

5. Fakruddin M, Mohammad Mazumdar R, Bin Mannan KS, Chowdhury A, Hossain MN.

Critical Factors Affecting the Success of Cloning, Expression, and Mass Production of Enzymes

by Recombinant E. coli. ISRN Biotechnol. 2012;2013:590587. doi: 10.5402/2013/590587.

PMID: 25969776; PMCID: PMC4403561.

6. Rosano GL, Ceccarelli EA. Recombinant protein expression in Escherichia coli: advances and challenges. Front Microbiol. 2014;5:172. doi: 10.3389/fmicb.2014.00172. PMID: 24860555; PMCID: PMC4029002.

7. Rosano GL, Morales ES, Ceccarelli EA. New tools for recombinant protein production in Escherichia coli: A 5-year update. Protein Sci. 2019;28(8):1412-1422. doi: 10.1002/pro.3668. PMID: 31219641 ; PMCID: PMC6635841.

8. Ki MR, Pack SP. Fusion tags to enhance heterologous protein expression. Appl Microbiol Biotechnol. 2020;104(6):2411-2425. doi: 10.1007/s00253-020-10402-8. PMID: 31993706.

9. Sinha R, Shukla P. Antimicrobial Peptides: Recent Insights on Biotechnological Interventions and Future Perspectives. Protein Pept Lett. 2019;26(2):79-87. doi: 10.2174/0929866525666181026160852. PMID: 30370841 ; PMCID: PMC6416458. 
10. Wang S, Zeng X, Yang Q, Qiao S. Antimicrobial Peptides as Potential Alternatives to Antibiotics in Food Animal Industry. Int J Mol Sci. 2016;17(5):603. doi: 10.3390/ijms17050603. PMID: 27153059; PMCID: PMC4881439.

11. Cui Y, Luo L, Wang X, Lu Y, Yi Y, Shan Y, Liu B, Zhou Y, Lü X. Mining, heterologous expression, purification, antibactericidal mechanism, and application of bacteriocins: A review. Compr Rev Food Sci Food Saf. 2021;20:863-899. doi: 10.1111/1541-4337.12658 PMID: 33443793

12. Sun P, Tropea JE, Waugh DS. Enhancing the solubility of recombinant proteins in Escherichia coli by using hexahistidine-tagged maltose-binding protein as a fusion partner. Methods Mol Biol. 2011;705:259-74. doi: 10.1007/978-1-61737-967-3_16. PMID: 21125392.

13. Waugh DS. The remarkable solubility-enhancing power of Escherichia coli maltose-binding protein. Postepy Biochem. 2016;62(3):377-382. PMID: 28132493.

14. Nguyen AN, Song JA, Nguyen MT, Do BH, Kwon GG, Park SS, Yoo J, Jang J, Jin J, Osborn MJ, Jang YJ, Thi Vu TT, Oh HB, Choe H. Prokaryotic soluble expression and purification of bioactive human fibroblast growth factor 21 using maltose-binding protein. Sci Rep. 2017;7(1):16139. doi: 10.1038/s41598-017-16167-x. PMID: 29170489; PMCID: PMC5700921.

15. Hanahan D. Studies on transformation of Escherichia coli with plasmids. J Mol Biol. 1983;166(4):557-80. doi: 10.1016/s0022-2836(83)80284-8. PMID: 6345791. 
16. Kojic M, Strahinic I, Fira D, Jovcic B, Topisirovic L. Plasmid content and bacteriocin

450 production by five strains of Lactococcus lactis isolated from semi-hard homemade cheese. Can

451 J Microbiol. 2006;52(11):1110-20. doi: 10.1139/w06-072. PMID: 17215903.

17. Miljkovic M, Uzelac G, Mirkovic N, Devescovi G, Diep DB, Venturi V, Kojic M. LsbB

Bacteriocin Interacts with the Third Transmembrane Domain of the YvjB Receptor. Appl

Environ Microbiol. 2016;82(17):5364-74. doi: 10.1128/AEM.01293-16. PMID: 27342562;

PMCID: PMC4988209.

18. Miljkovic M, Lozo J, Mirkovic N, O'Connor PM, Malesevic M, Jovcic B, Cotter PD, Kojic

M. Functional Characterization of the Lactolisterin BU Gene Cluster of Lactococcus lactis subsp. lactis BGBU1-4. Front Microbiol. 2018;9:2774. doi: 10.3389/fmicb.2018.02774. PMID:

30498487; PMCID: PMC6249370.

462

19. Vukotic G, Mirkovic N, Jovcic B, Miljkovic M, Strahinic I, Fira D, Radulovic Z, Kojic M.

464 Proteinase PrtP impairs lactococcin LcnB activity in Lactococcus lactis BGMN1-501: new 465 insights into bacteriocin regulation. Front Microbiol. 2015;6:92. doi: 466 10.3389/fmicb.2015.00092. PMID: 25713574; PMCID: PMC4322719.

469 raffinolactis BGTRK10-1, an Isoschizomer of EcoRI, Exhibits Ion Concentration-Dependent

470 Specific Star Activity. Biomed Res Int. 2018;2018:5657085. doi: 10.1155/2018/5657085. 
bioRxiv preprint doi: https://doi org/10.1101/2021.05.26.445771: this version posted May 26.2021 . The copyright holder for this preprint

(which was not certified by peer review) is the author/funder, who has granted bioRxiv a license to display the preprint in perpetuity. It is made available under aCC-BY 4.0 International license.

473 21. Malesevic M, Stanisavljevic N, Miljkovic M, Jovcic B, Filipic B, Studholme DJ, Kojic M.

474 The large plasmidome of Lactococcus lactis subsp. lactis bv. diacetylactis S50 confers its

475 biotechnological properties. Int J Food Microbiol. 2021;337:108935. doi:

476 10.1016/j.ijfoodmicro.2020.108935. PMID: 33152568.

477

478 22. Raran-Kurussi S, Waugh DS. Expression and Purification of Recombinant Proteins in 479 Escherichia coli with a His ${ }_{6}$ or Dual His ${ }_{6}$-MBP Tag. Methods Mol Biol. 2017; 1607:1-15. doi:

23. Bucher D, Grant BJ, McCammon JA. Induced fit or conformational selection? The role of

483 the semi-closed state in the maltose binding protein. Biochemistry. 2011;50(48):10530-9. doi:

484 10.1021/bi201481a. PMID: 22050600; PMCID: PMC3226325. 


\section{Supporting information}

S1 Fig. SDS-PAGE analysis of MBP-His 6 -LenA recombinant fusion protein purified via

(A) Ni-NTA agarose or (B) amylose resin. Lane 1: flow-through proteins, Lane 2: proteins

removed by wash 1, Lane 3: proteins removed by wash 2, Lane 4: proteins removed by wash 3,

Lane 5: eluate1, Lane 6: eluate 2, Lane 7: eluate 3, Lane M: protein molecular weight marker

(BlueEasy Prestained Protein Marker). Black arrow indicates eluted overexpressed MBP-His 6 -

S2 Fig. SDS-PAGE analysis of MBP-His $_{6}-\mathrm{hBD}$ recombinant fusion protein purified via

(A) Ni-NTA agarose and (B) amylose resin. Lane 1: flow-through proteins, Lane 2: proteins

removed by wash 1, Lane 3: proteins removed by wash 2, Lane 4: proteins removed by wash 3,

501 Lane 5: eluate 1, Lane 6: eluate 2, Lane 7: eluate 3, Lane M: protein molecular weight marker

(BlueEasy Prestained Protein Marker). Black arrow indicates eluted overexpressed MBP-His $6^{-}$ hBD fusion AMP.

S3 Fig. The cleavage efficacy of enterokinase depends on MBP-His6-LenA purification

method. SDS-PAGE analysis of MBP-His ${ }_{6}$-LcnA recombinant fusion protein after cleavage by

506 His $_{6}$-enterokinase. The MBP-His 6 -LcnA fusion protein purified using Ni-NTA agarose resin

507 (Lane 1) or amylose resin (Lane 2) and cleaved by His tagged entrokinase for 3 h. Lane M:

508 protein molecular weight marker (BlueEasy Prestained Protein Marker). Black arrows indicate

509 the products of enterokinase digestion. Open framed arrow indicates the position of undigested

510 fusion tags-AMP. 
pMALc/p5HisEk MCS
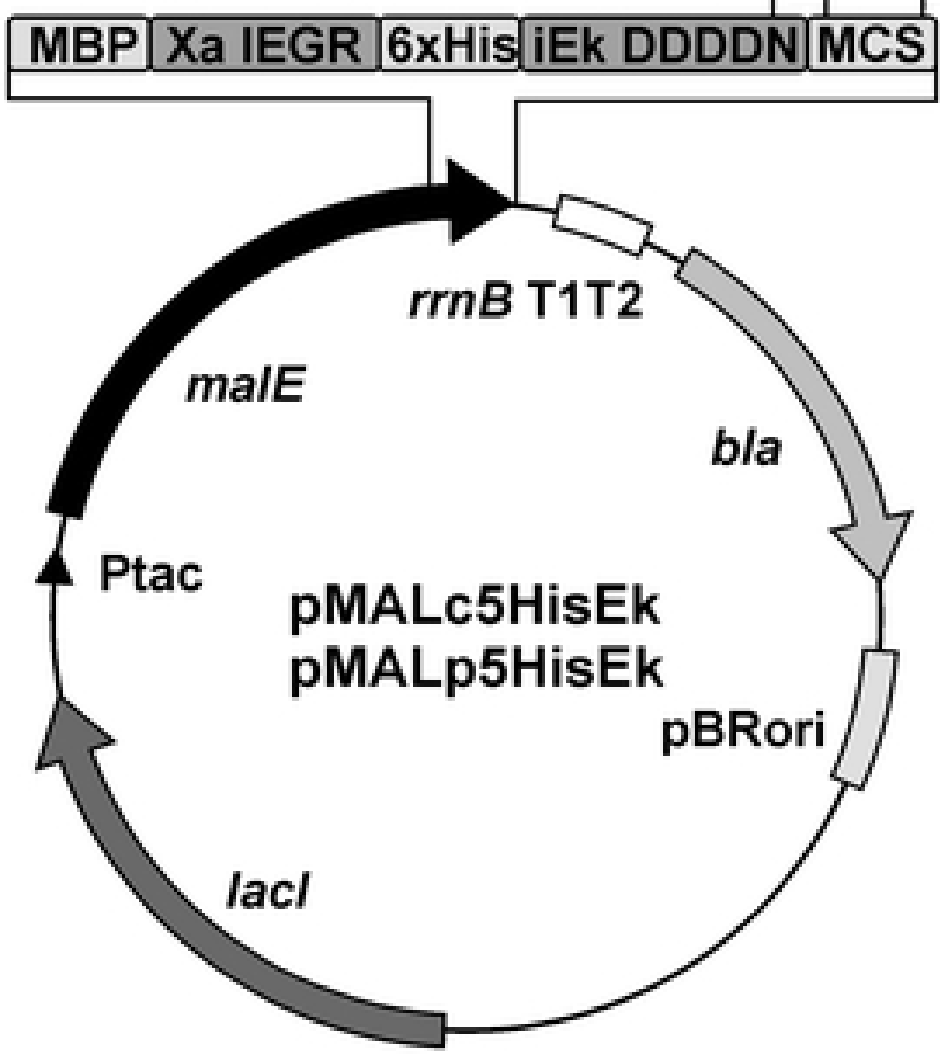

Sacl

TCGAGCTCGAACAACAACAACAATAACAATAACAACAACCTCGGGATCGAGGGAAGGCATCACCATCACCATCAC

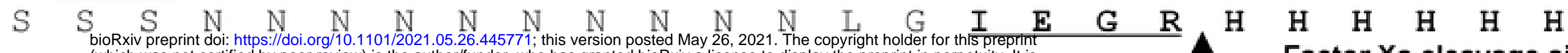

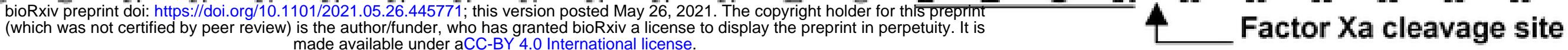

BsaBl Sphl Sacl Kpnl Ndel Ncol Eagl EcoRV
GGATCTGATGATGATGATATTATCGCATGCGAGCTCGGTACCCCATTTCACATATGTCCATGGGCGGCCGCGAT

\begin{tabular}{lllllll|llllllllllllllllllll}
$G$ & $S$ & $D$ & $D$ & $D$ & $D$ & $N$ & $Y$ & $R$ & $M$ & $R$ & $A$ & $R$ & $Y$ & $P$ & $I$ & $S$ & $H$ & $M$ & $S$ & $M$ & $G$ & $G$ & $R$ & $D$
\end{tabular}

Sall

BsaBI cleavage blunt position

Hincll

Accl BamHI EcoRI Pstl HindIII

ATCGTCGACGGATCCGAATTCCCTGCAGGTAATTAAATAAGCTTCAAA

$\begin{array}{llllllllllllllllllllllll}I & V & D & G & S & E & F & P & A & G & N & * & I & S & F & K\end{array}$

B

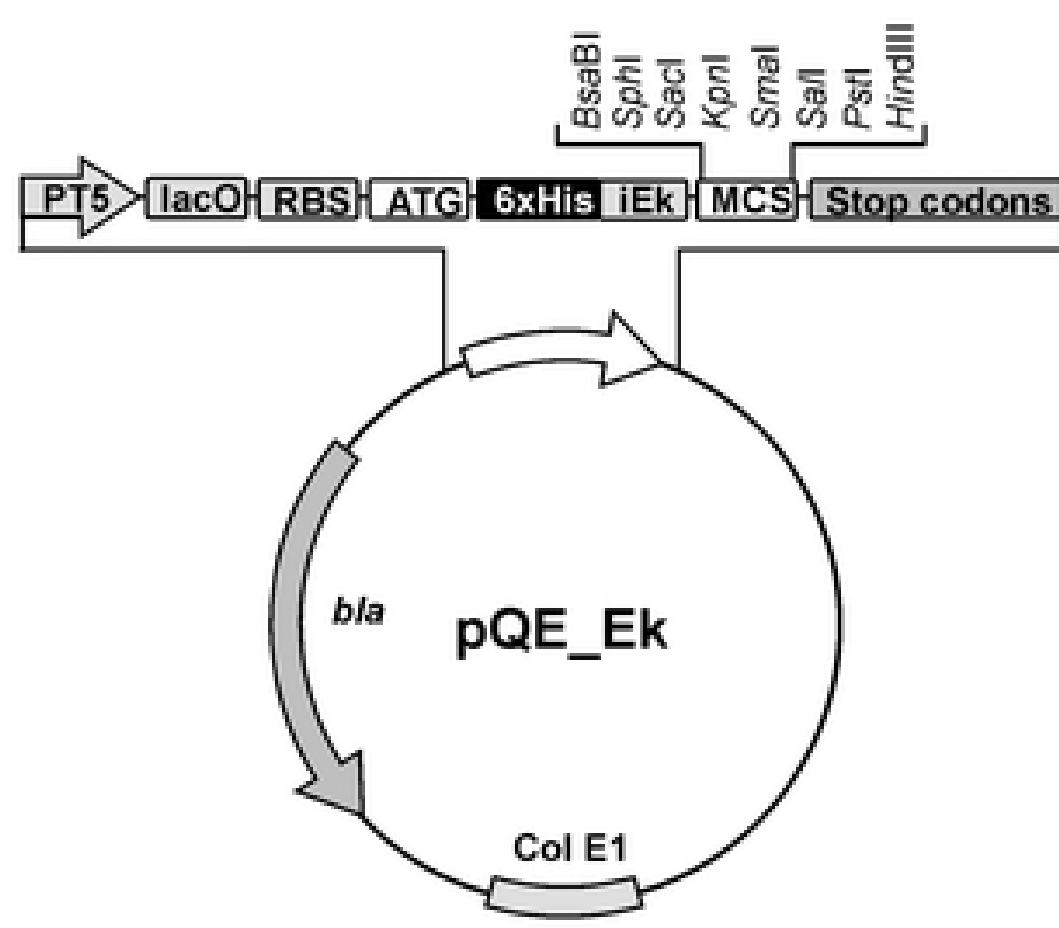

EcoRI

GAATTCATTAAAGAGGAGAAATTAACTATGAGAGGATCGCATCACCATCACCATCACGGATCTGATGATGAT

$\begin{array}{llllllllllllllll}M & R & G & S & H & H & H & H & H & H & G & S & D & D & D\end{array}$

Sall

Hincll

BsaBI Sphl Sacl Kpnl Smal Accl Pstl HindIII

GATAATtATCGCATGCGAGCTCGGTACCCCGGGTCGACCTGCAGCCAAGCTTAA

\begin{tabular}{ll|llllllllllllllll}
\hline & N & Y & R & $M$ & R & A & R & Y & P & $G$ & S & T & C & S & $Q$ & $A$ & *
\end{tabular}

BsaBI cleavage blunt position

Figure 1 
A

1

$\begin{array}{lllll}2 & 3 & 4 & M & 1\end{array}$

12

3

4

$\mathrm{kDa}$

bioRxiv preprint doi: https://doi.org/10.1101/2021.05.26.445771; this version posted May 26, 2021. The copyright holder for this preprin

made available under aCC-BY 4.0 International license.

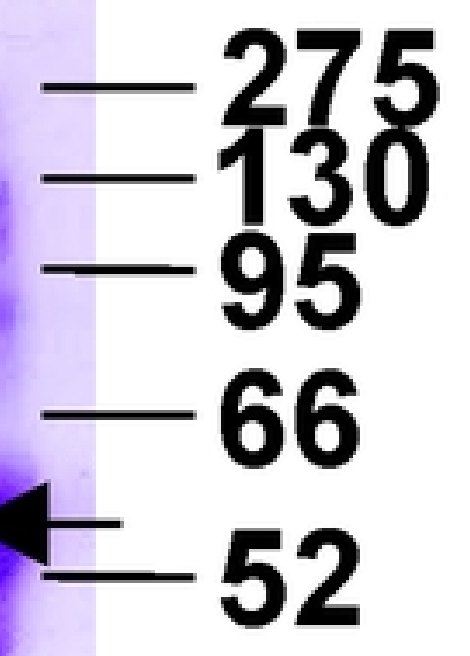

$-37$

$-30$

16

$-6.5$

Figure 2 
A

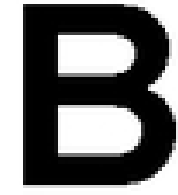

$\begin{array}{llllll}1 & 2 & 3 & 1 & 2 & 3\end{array}$

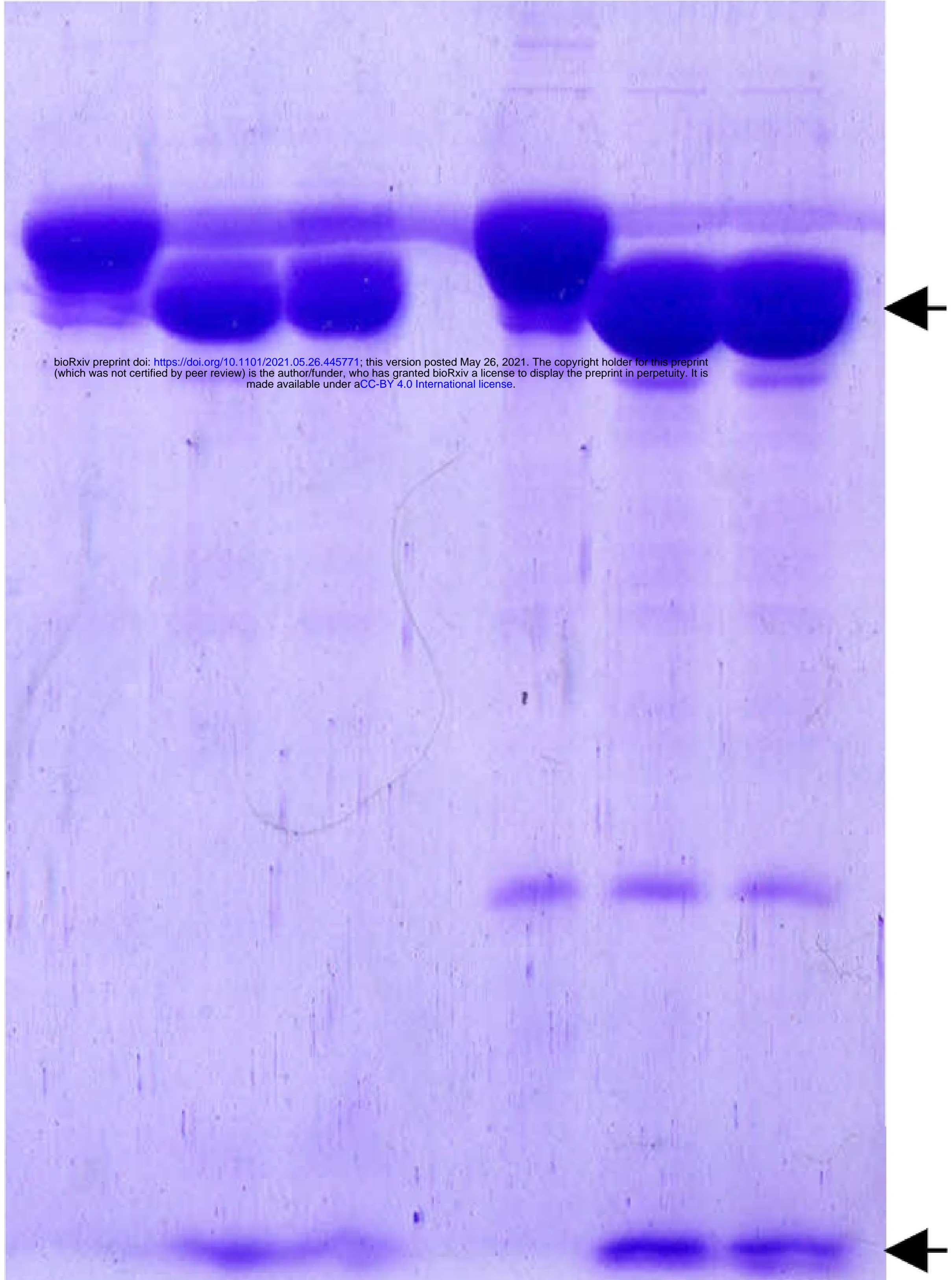

Figure 3 
$A$

$\begin{array}{llllll}1 & 2 & 3 & 4 & 5 & M\end{array}$

$\begin{array}{llll}1 & 2 & 3 & 4\end{array}$

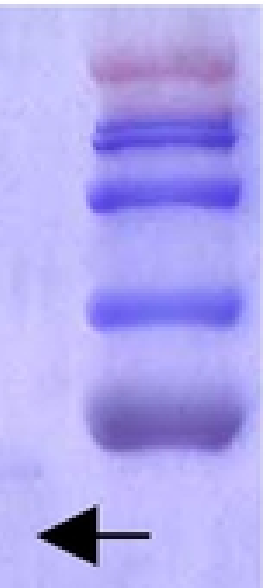

$\Rightarrow$

$\Rightarrow$

16

$-6.5$

4

Figure 4 


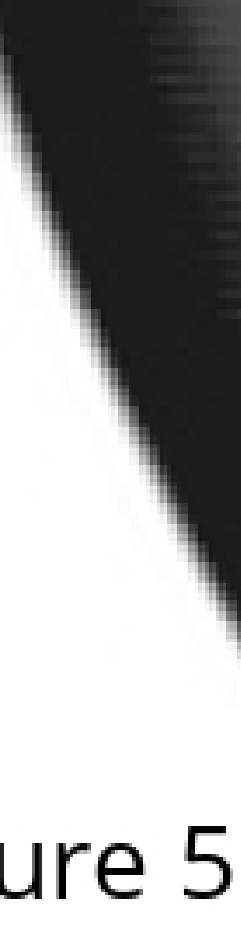

hBD

Figure 5 
Double digestion (BsaBI-X) of vector (pMALc/p5HisEk)
PCR amplification of gene encoding protein/peptide with added A at $5^{\circ}$ end

Digestion of PCR with "X" $R$. enzyme

Ligation

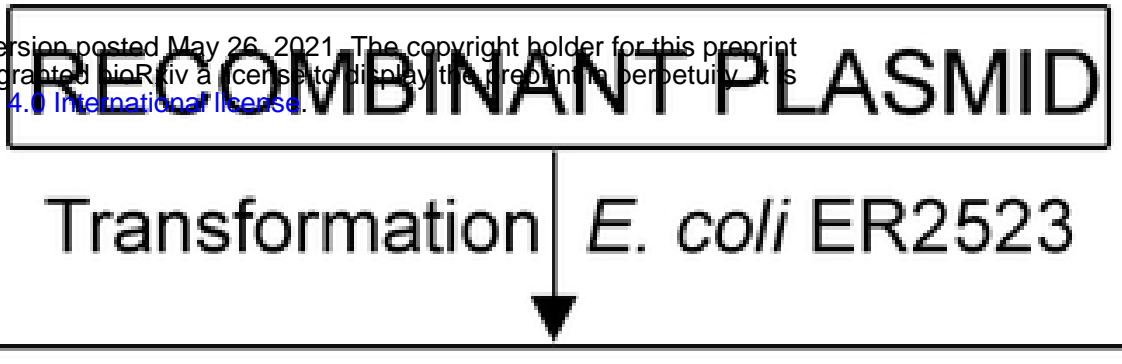

Transformants with pMALc/p5HisEk-P clone

$\mathrm{PCR}, \mathrm{RE}$ analysis confirmation by sequencing

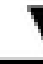

Induction of $\mathrm{MBPHis}_{6}-\mathrm{P}$ expression

SDS-PAGE analysis

Purification of $\mathrm{MBPHis}_{6}-\mathrm{P}$ fusion protein using Ni-NTA agarose

Buffer exchange

Cleavage with enterokinase

SDS-PAGE analysis

Removal of $\mathrm{MBPHis}_{6}$ tag by amylose resin and enterokinase by Ni-NTA agarose

\section{PURE NATIVE PROTEIN}

SDS-PAGE analysis

Activity analysis

Figure 6 